lohe Vorgang der Materialprüfung und es wiŕd bei der Untersuchung oine der Wirklioh. keit mögliohst angenäherte Belastang hergestellt, Im Flugzengbau wird aber, hanpt" shchlich wegen der großen Unsicherheit in den Annahmen über die Materialkonstanten, die zulässigen Belastungen usw, auch eine Bruchprobe des.ganzen Tragwerks, d. $h$. der Zelle und der Pyramide, vorgenommen, ein Versuch, der zugleich eine Kontrolle fär, die Riohtigkeit der Festigkeitsrechnung bildet. Gewöhnlich wird die Belastungsprobe für den Steigflug ausgeführt ${ }^{1}$ ).

Wenn man vom gesamten Filugzenggewicht $G^{\prime}$ das Gewicht $G_{1}$ der Tragzelle abzieht, so erhalt man, wie eingangs beschrieben, die beim normalen Flug die Zello angreifende Last. Man wendet nun das Flugzeug um, anterstützt den Rumpl añ den Stel. len, wo im Betrieb die gröBten Laften, wie Motor, Bemannung and Benzinvorrat liegen, so daß bei Belastung im Rumpf eine ahnliche Beanepruchung wie im Flug (d. h. ein ahnHoher Verlauf der Biegungsmomente und Querkräfte) hervorgernfen wird: Erreicht wird des einfach durch Anwendung eines Satzes von Wagebalken(Balanoe)trägern; zweiarmige Hebel bzw. einfache Balken iibertragen die Unterstiutzungskräfte in statisch bestimmter Weise. Bei der Bruchprobe werden die jetzt nach oben gekehrten Flügelunterseiten mir Gewichten, gewöhnlich mit Sand belastet. $U m$ den Zustand für normalen Steigflug zu erreichen, ist insgesamt ein Gewicht von $G-2 G_{1}$ aufzabringen, da das Gewicht $G_{1}$ der Zelle im Sinne der Belastang wirkt. Bei der in der Regel für Steigflug geforderten fünffachen Bausicherheit darf also die Konstruktion erst bei einer. Sandlast von $5 G-6 G_{1}$ ihre Tragfähigkeit verlieren.

Die Herstellung eines Flugzeuges ist heute fast ausschlieBlich eine Konstruktionsangelegenheit geworden. Ebenso wie bei Tragwerken des Hoch-oder Brückenbàues wird auf Grund der statischen Berechnang eine Dimensioniernng der einzelnen Organe vorgenommen. Beim Bau gröBerer Flugzeuge tritt die Notwendigkeit der BachgemäBen Berechnung auch augenfällig zutage. Wir wollen jedoch hiermit keineswegs die Rolle der Statik tuberschätzen. Nur die richtige Umfetzung aeromechanischer Erkenntnisse in die Praxis, der Ban leichter Motore und insbesonders Ausdauer und Entschlossenheit mutiger Flieger baben es bewinkt, daßs "zu deE, Geistes Flügeln auch körperliche Flügel sich gesellen “. 3

\title{
Über neuere Lehrbücher der praktischen Analysis.
}

von L. BIEBERBACH in Frankfurt a. M.

$\mathrm{L}$ abit man das Auge iiber die Titel der im vergangenen Jabrzehnt erschienenen Lebrbücher aus dem Gebiete der praktischen Mathematik gleiten "), so fällt auf den ersten Blick das Torwiegen des Graphischen auf. Graphische Methoden nennt denn anch Runge, dem das ganz neue Gebiet ein gat Teil seines Lebensbewnßtseins verdankt, sein jetzt in zweiter deutscher Auflage vorliegendes Buch, das aus in Amerika gehaltenen Vor trägen hervorgegangen ist. Leitfaden zum graphischen Rechuen nennt Mebmke. - eln in der gleichen Teubnerschen Sammlung mathematischer uvd physikalischer Schriften fulr Studierende und Ingenieure erschienenes Werk. Ueber graphische Integration sohreibt Willers, einer der leider' wenigen auf unserm Gebiete bewanderten Oberlehrer, oln Bundchen für die Sammlung Goeschen. Praktische Analjsisc freilich nennt der Vertreter der Mathematik an der Bergakademie in Clausthal, v. Sanden, sein Büchlein, das gleichfalls im Teubrierschen Verlag erschienen ist; doch schon ein Blick aufs Inhaltsverzeichnis läßt erkennen, daß der Verfasser trotz seines Strebens gufs Ganze das Graphlsohe nicht weniger betont als die iibrigen Autoren. Neben diesem Vorwiegen des Graphischen fält die bewubte Einstellung anf die Probleme der Analysie sofort in die Augen, sowie man sur. einen Vergleich mit den nur wenig älteren Lehrbüchein von Blermann, Bruns, Lüroth ïber numerisches Rechnen versucbt. Die Methoden der meohanischen Quadratur sind das einzige, was dort zur Bewältigung der Probleme vor-

1) Saliger, Vortrage Uber Statlk des Flugzeugbaues. k. u. k. Fliegerarsenal, wien 1916. Hoff, Verfabren bel Flugelfestigkeiteprufungen, Zettschr. f. Flugt. und Motorl., 1916.

, 2) C: Runge, Graphische Methoden, 2. Aufl., Leipzig 1019; R. Mehmke, Leitfaden zum graph1. wohen Rechnen, Lelpalg 1917; A. Willers, Grapheche Integration, Lelpzig 1920; H. v. Sanden, 'rraktiuche Analysis, Lelpzig 1914. 
getragen wird, welche die Mittel der Analysis verlangen. Geht man' im Vergleioh mehr aufs Einzelne, so wird man sofort hinsichtlich der Answahl von Vergleichspnnkten in Verlegenheit kommen. Damit wird nicht béstritten, daB Vorläufer im einzelnen tär den Gegenstand der genannten Werke da sind, aber bewußt nou ist dio Verarbeitung der Einzelheiten zu einem neuon ausichtovollen Gobiet mathematiseher. Forschung; bewubt neu lst die Tendenz: die Durcharbeitung der reln mathematischen Methodon bis zur praktiochen Brauchbarkeit. ... .

Dem Zurüokgehen auf den' Ausgangspunkt, wie es Felix Kloin seit fast 40 Jahren verlangt, verdankt die nene Richtang ihr Leben. Dis Praxis in Physlk, Technik: usw... stellt der mathematischen Forschung bestimmte Probleme. Aber Steine statt Brot erhlelt sie meist von dieser als Gegengabe. Es muB gehen, sagen die reinen Mathematiker, aber fragt mlch nur nioht wie, mir genügt es zu wisen, daB es geht. Da setzt die nene Rich? tang ein. Sie besityt zu viel Disziplin, um sich mit 60 oberflächlioher Antwort zu begnit gen. Sie will aus der potentiellen eine aktuelle Möglichkeit machen und de mathematischen Methoden wirklioh to durobarbeiten, dab man sie handhaben kann, und das man das numerische Ergebnis erzielen kann, an dem der Praxis gelegen ist.

1. Gleichungs-Auflösung. Ich will an ein paar Beispielen zeigen, wio die Móderne hier ihrer Aufgabe gerecht wird, Ich beginne mit einem Problem der Algebra. Wie löst man eine Gleichung dritten Grades auf?. Wer efnen schlechten Unterrioht auf Schule oder Hochschule genossen hat, dem 'fallt, wenn überhaupt etwas, so in eriter Linie die Cardanische Formel eln. Betrachten wir z. B. die Gleichnng

$$
x^{3}+2,1 \cdot x^{2}+3,4 \cdot x-1,7=0 \quad . \quad . \quad \therefore(1) .
$$

Will man die Cardanfsche Formel anwenden, so hat man zanächst durch die Substitution

$$
\text { enr Gleichung } \quad, \quad x=z-0,7
$$

iberzugehen. Will man die reelle Warzel nun wirklich auf drei Dezimalen genau bèstimmen, so wird man auf die dritte Stelle binter dem Komma in dem letzten Koeffizienten nicht verzichten dürien. Rechnet man nach der Vorschrift der Cardanischen Formel, so bestimmt man zunächst

$$
\begin{array}{lc} 
& \frac{(3,394)^{2}}{4}+(1,93)^{3}=3,146, \\
\text { alsdann } & \sqrt{3,146}=1,774, \\
\text { ferner } & \frac{3,394}{2} \pm 1,774= \pm 3,471 \\
\text { endlich } & z=\sqrt[3]{3,471}-\sqrt{0,077}=1,089 \pm \frac{1}{.10^{3}}
\end{array}
$$

Also ist

$$
x=0,389 \pm \frac{1}{10^{3}}
$$

Wenn man auch vielleicht auf trigonometrisohem Wege diese Rechnung ein weñig abkürzen kann, so bleibt doch die Frage offen, ob die Cardanische Formel nicht durch eine bessere Jechenvorschrift ersetzit werden kann. Denn es ist dooh auffillig, dab man erst eine gewisse vielleicht vermeidbare Rechenarbeit durch die Aufstellang der Formel ein für allemal leisten mufs. Könnte man nicht mit dem Berechnen der Wurzel unmittel: bar an die Gleichung anknïpfen? Das geht in der 'Tat und die Erlédignng dieser Frage hat zu sehr brauchbaren Methoden der Auflösung geführt. Der Gedanke, welcher der besten Methode zugrunde liegt, ist sehr einfach. Man bewalligt das Problem durch rationelles Probieren.' Das Prinzip des Probierens aber ist dieses: Merkt man beim Einsetzen zweier verschiedener $x$-Werte in die Gleichung, daB ihre linke Seite Werte von verschledenem Vorzeichen erhalt, so weiß man, daß zwischen beiden $x$-Werten Warzeln liegen müssen. Man wird nuv bestrebt sein, duroh Fortsetzung des Problerveriatrens die Wrrzeln in immer engere Grenzen einzuschließen, bis man die gew ünsohte Genaúigkeit erreicht hat.

Dies Probieren kann langwierig sein, wenn man nicht 1) die dazu nötigen Rechnungen mögllohst geschickt und rasch auszuführen versteht, wenn man 2) dio versuchinweise anzajetzenden Werte nicht nach vernünftigen Gesichtspnnkten answahlt 
Die Rechnungen werden am bequemsten nach dem Hornerschen Schema augge, füht, d. h. man rechnet so, wie es die folgende Schreibweise andeutet:

$$
f(x)=x^{3}+2,1 x^{2}+3,4 x-1,7=[(x+2,1) x+3,4] x-1 ; 7 .
$$

Am üborsichuichsten notiert man slch das so:

\begin{tabular}{c|c|c|c}
1 & 2,1 & 3,4 & $-1,7$ \\
\hline & $1 \cdot x$ & $(2,1+x) x$ & $x[(2,1+x) x+3,4]$ \\
\hline & $2,1+x$ & $(2,1+x) x+3,4$ & $f(x)$
\end{tabular}

Diese Art des Rechens bietet namentlich bei. Verwendung des Rechenschiobers den ersichtlichen. Vorteil, daß man mit ein und derselben Zungen- stellung auskommt.

Die Auswahl der zum J'robieren heranzuziehenden $x$-Werte gelingt am besten, wenn man die eben angegebene Rechnung graphisch ausführt, weil man da am besten den Linflue einer' $x$ - $\Lambda$ enderang anf den Funktionswert $z u$ beurteilen vermag. So gelangt man zu dem bewährten Lillschen Rechtwinkelverfahren, dem ich vor andern gleichfalls möglichen den Vorzug geben möchte.

Man trägt die Koeffizienten in irgend einer Ginheit in oinen reehtwinkligen Streckenzug ab derart, das jede Strecke einen Koefii:ienten zur MaBzahl hat und das man beim Uebergang zum folgenden Koeffizienten eine liechts- oder eine linksschwenkang auszuführen hat, jo nachdem, ob beide gleiches oder verschiedenes Vorzejehen haben. Fïr ${ }^{*}$ unsere Gl. (1) kommt so Alb. I zum Vorschein.

$$
\text { Nun wäblen wir einen }
$$
beliebigen $x$-Wert $\left(x_{0}\right)$ aus, den wir in der gewällten Winheit auf der aus $A \mathrm{bb}$.: ersfchtlichen $r$-Skala markieren. $x+y, 1$ ist nun sofort in der Abbildung als vertikale Strecke $Q C$ zu bemerken. Nun gehen wir senkrecht $\% u$ der Verbindungslinie $A Q$ nach $l l$ auf der Geraden $D C$. $C R$ hat dann die Länge $(x+2,1) x$, weil die beiden Dreiecke $A B Q$ and $Q C R$ lihnlioh sind. $D C$ entspricht. dann $x(x+2,1)+3,4$. Nun hat man noch ein letztes $\mathrm{Mal}$ mit $x$ zu multiplizieren. Dazu geht man wieder senkrecht $z u$ $Q R$ vor bis nach $S$ auf der letzten Koeffizientengeraden. Auf der $y$-Skala liest man dann den $x_{0}$ entsprechenden Funktionswert ab. Wünscht man also eine Wurzel. der Gleiohung zu bestimmen, so muB man $x_{0}$ so wählen, daB $S$ mit $E$ zasammenfullt. Das muB man duroh Probieren $z \mathrm{n}$ orrelchen suchen.

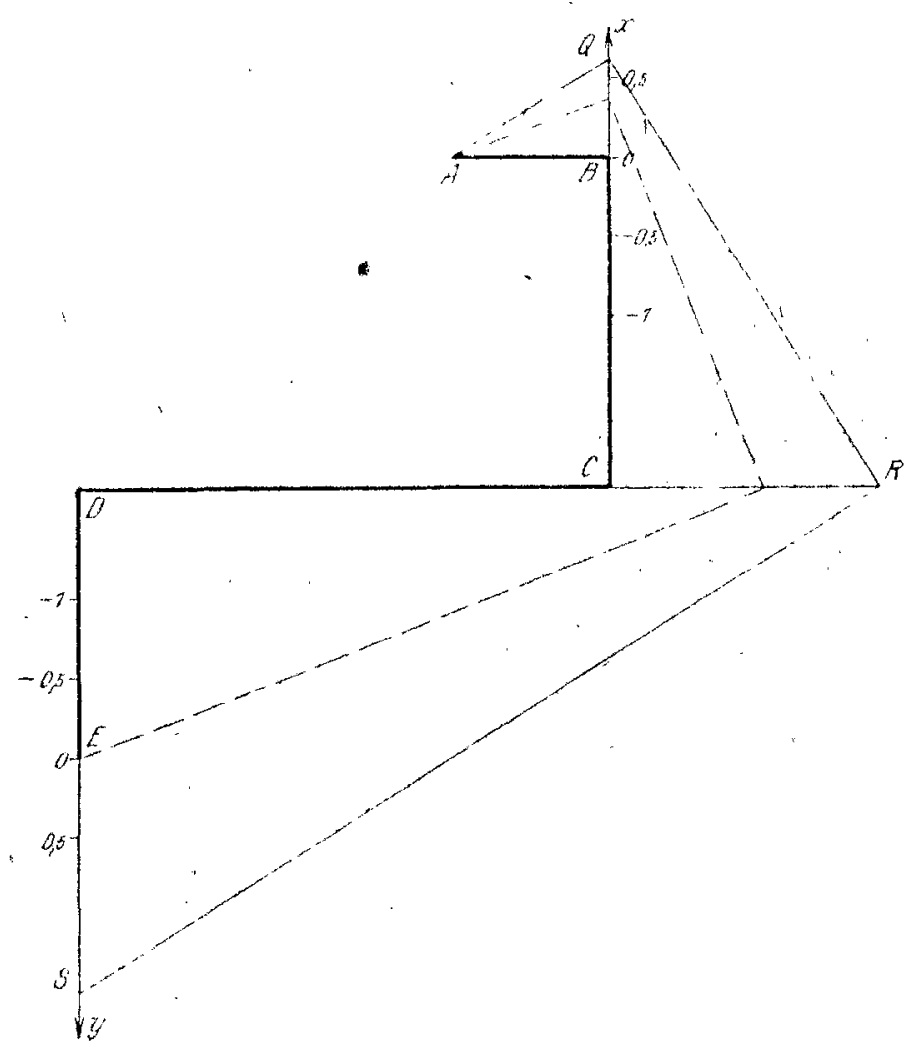

Abb. 2 
- Fis ist nun aber das Bereohnen des Funktionswertes, welcher elnem ansgewahlten $x$-Wert entspricht, so einfach und tibersichtlich geworden, daB man in kürzester Frist ans freier Hand skizzierend eine grobe Zahl von ungefähren Proben machen kann. Hat man dann mit eíniger Sicherheit eine Wurzel gefunden, só wird man sorgfaltiger zeiohnen und die Genauigkeit erhöhen. Bei der Ansfübrang bietet es erhebliche Frleichterung, wenn man eine Millimetereinteilung ans durchscheinendem Papier benutzt. Dies Blatt bewegt man dayn über dem. Blatt, anf dem der Koeffizientenzag verzeichnet ist, und verfolgt den Rechnungszang längs der Einteilangslinien des 'durchscheinenden Blattes. Hat man so die Wurzel mit der mögliohen Genauigkeit festgestellt, so kennt man ein enges Intervall, dem sie angehört. Dann kann man leicht die Regula falsi verwenden, um eine weitere Verbesserung za erzielen. Den lösënden Zag unseres Beispjeles haben wir in Abb. 2 gestrichelt eingezeichnet. Man liest $x=0,38$ ab. Rochnet man den zugehörigen Fanktionswert, näb dem Hornerschen Schema aus, so findet man $-0,049888$. Der Wurzelwert ist also zu klein. Für $x=0,4$ abér erhält man nach der Zeicbnung einen deutlich positiven Wert, den man zu 11,16 berechnet. Also liegt die Wurzel zwischen 0,38 und 0,4 . Wir haben so wesentlich bequemer dieeslbe Genauigkeit wie bei der Cardanischen Formel erreicht. Die Regula falsi llefert als neuen Näberungswert

Nan findet $f\left(r_{1}\right)=-0,0002$.

$$
x_{1}=0,38+0,02 \cdot \frac{0,049888}{0,109888}=0,3891
$$

Ueber das Lillsche Vesfahren, dessen besonderer Vorzug auch die unveränderte Anwendbarkeit aut Gleichungen beliebigen anderen Grades ist, mag man sich noch nalher in den genannten Büchern informieren oder auch in Mehmkes Artikel im ersten Band der Enzyklophdie der mathematischen Wissenscheften, oder auch in Scheffers Lehrbuch der Mathematik. Dagegen wird man in den auf technischen Hocbschulen und Universitäten üblichen einführenden Büchern iiber höhere Mathematik oder Algebra leider vergeblich nach dem Verfahron suchen.

In den erwähnten Werken wird man noch manche andere Metboden finden. Diese laufen anf ein Aufzoichnen der Kurve

$$
y=x^{3}+2,1 x^{2}+3,4 x-1,7
$$

in rechtwinkligen Koordinaten hinaus. Einige Vorteile bietet dabei die Verwendung logarithmisch geteilten I'apiers, wie man das bei der Lektüre des Mehmkeschen Buches bemerken wird. Jedenfalls aber verlangt dies logarithmische Verfahren Mehmkes darüber hinaus besondere Zurichtungen (Additionslogarithmen), während das Lillsche Verfahren ohne weiteres anwendbar ist.

Genügt die hiermit zu erzielende Auflösungsgeschwindigkeit noch nicht allen Ansprüchen, oder hat man sehr oft Gleichungen gleicher Bauart aufzulösen, möchte man die Wurzeln ebenso rasch und bequem finden, wie man etwa einen Logarithmus aufschligt, so muB man eine Tatel, ein Nomogramm der Gleichungen dritten Grades, herstellen. Die besten beruhen auf folgendem Prinzip:

Wir legen eine gerade Linio durch ihre Abschnitte $u, v$ auf zwei parallelen Geraden fest. Wir nennen die $" v$ Koordinaten der geraden linie.' Einer linearen Glej-

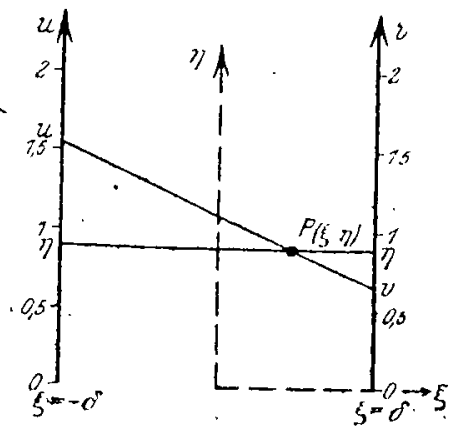

Abb. 8 chung $z$ wischen $u$ und $v$ entspricht dann eine Schar von geraden linien. Sieht man naher za, so bemerkt man, daß die Geraden alle durch oinen Punkt gehen, der dann also durch die lineare Gleichung bestimmt ist; diese heiBt dann Gleichung des Punk'es.'

Um die Behauptung einzusehen, braucht man nur einen Blick auf die beiden ähnlichen Dreiecke der Abbildung 3 zu werfen. Dann liest man sofort die Proportion

ab. Daraus aber folgt

$$
\frac{\eta-v}{\eta-u}=\frac{\xi-\delta}{\xi-\delta}
$$

$$
v(\zeta+\delta)+u(\delta-\xi)-2 \eta \delta=0
$$

als Bedingung dafür, daß eine Gerade mit den Koordinaten $u$ und $v$ 'durch den Punkt $P$ mit den rechtwinkligen

Koordinaten $(\xi, \eta)$ geht. Vergleicht man dête gefurdene mit einer beliebigen linearen Glejohung

$$
a v+b u+c=0 . \therefore . . . . . .(3),
$$


10 arblt man die Proportion

$$
a: b: c=\xi+\delta: 8 \div \xi:-2 \eta \delta
$$

wad daran findet man, daB Gl. (3) de Gleichung des Panktes

Ith. Die knbisohe Gleiohung 1.

$$
\xi=\delta \frac{a+b}{a+b}, \eta=-\frac{c}{a+b}
$$

$$
x^{3}+u x+v=0
$$

donken wr uns duroh eine Gerade mit den Koordinaten $u$ und $v$ dargestellt, Söll diese Ololohang oine Wurzel $\dot{x}_{0}$ besitzen, so mas die Gerade duroh den Pankt $P_{0} ;: \cdots$

$$
\xi=\delta \frac{1-x_{0}}{1+x_{0}}, \eta=\frac{-x_{0} a^{2}}{1+x_{0}} \cdots \quad: \quad \because \because \because \because \cdots
$$

mben. Allen knbischen Gleiohnngen mit der Wurzel $x_{0}$ entspreohen also gerade Linien duroh dlesen Pankt, and wenn elne Gorade durch diesen Pankt geht, so hat eben die lhr entuprechende kubische Gleichung die Wurzel $x_{0}$. Dlo den verschiedenen Wurzeln entsprechenden Huakte kann man aber eln für allemal markieren. Alo gebrrer alle einer Kurvo an, die in G!. (5) In Jarameterdarstellung angegeben ist. Ihr den vonlllven $x$-Werten enteprechender Ast ist in $\mathrm{Abb}$ : anntbernd angedeutet. Eine vollstindige, maßHabrlobtlge Ansfïhrang dieser Zeichnung - stellt ans Nomogramm der dreigliedrigen kubischen Clolchang (4) dar.

Will man auch negative Wurzeln mit Hilfe dos Nomogramms bestimmen, so braucht man nur In dor Glelohung (4) $x$ durch $-x$ zu ersetzen. Juroh eine gestrichelte Linie in Abb. 4 habe ich dlo Gleichung (2), die uns trüher beschäftigte, ungrodeutet.

Das geschilderte Verfabren läßt sich natürllob anf belieblge Gleichangen der Gestalt $f(x)+u g(x)+v h(x)=0$ ausdehnen. Aber an do ullgemeine Gleiohung

$$
x^{3}+u x^{2}+v x+w=0
$$

Arltton Grades kommt man so nicht heran. Will man so nomographisch darstellen, so muß man ontwoder eine Umrechnung vornehmen aut eine Alolohung mit nur zwei willkürlichen KoeffiMlenten, etwa durch Wegschaffen des quadratiwohen Gliedes, oder man muß sich fïr jeden Wert von $w$ elne besondere Lösungskurve zelchnen. XMIn derartiges Nomogramm findet der Leser in Mobmkes Artikel im ersten Bande der mathemalluchen Enz̧klopädie.

Die hiermit knapp angedeutete Nomopruphie bat sioh in den wenigen Jahrzehnten ibres

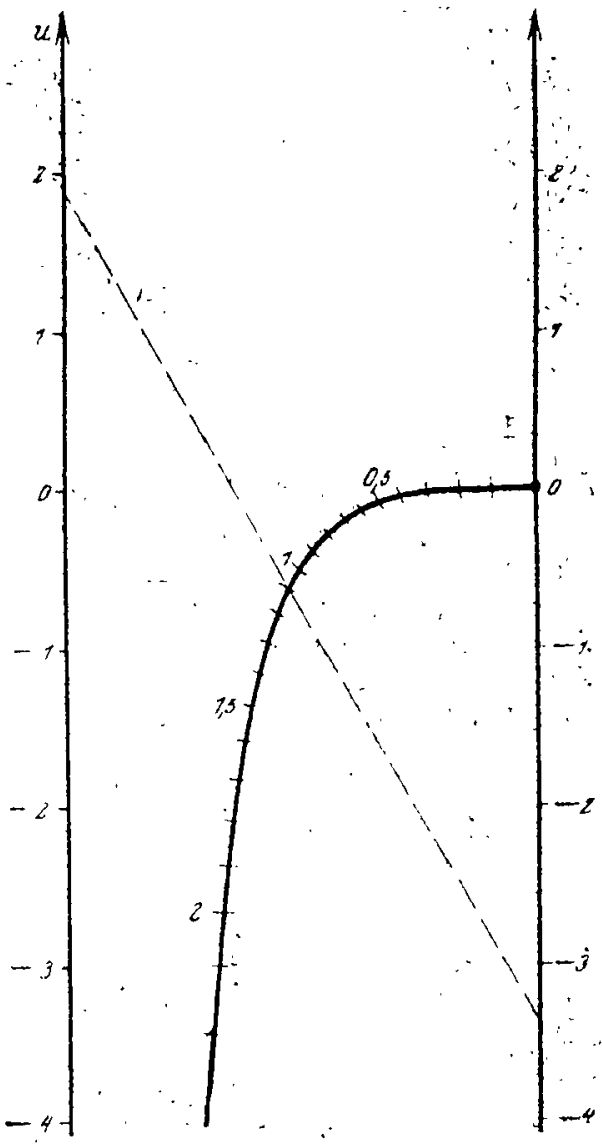

Abb, 4 Bontohens zu einem der an Anwendungen reichstèn Vorfebren der graphischen Darstellung entwickelt. Ueber weitere Einzelheiten dieser wlobtlgen Methode wird der Leser sich an Hand der erwllhnten Literator leloht weiter Informieren, wenn er nicht zu D'Ocagnes grobem Traité de nomographie oder zu Goblllings, bei Te uber erschionenem Auszug, oder zu Luckeys kurzer Darstellung In Teubners mathematischer Bibliothek greifen will. Wieder aber ist za bedapern, dab dio jm Mittelpunkt des mathematischen Hochschulinterrichts stebenden Werke'so gar keine Ruoksioht auf diese für den Schulunterricht doch schon so geeignelen Dinge nehmen. $\because$

2. Differentialgleichungen. Die Tendenz, die matticmatischen Methödon bis sut praktischen Brauchbarkeit durchzabilden, hat in neuester Zeit anch in Gebiete der unperen Analy sis Erfolge zu zeiligen begonnen. Freilich sind nooh lange nioht alle, pioleobr nur die allerwenigsten Probleme der Analysis im Besitze einer wirklfoh fertig 
durcbgebildeten Methodik. In besonders glücklicher Lage ist man durch Runge in der Theorie der Differentialgleichungen, insbesondere bei den gewöhnlichen: Die Problemlage ist hier Uhnlich wie in der Algebra. Man verfügt seit Alters über geschlossene Integrationen, über Existenztheoreme, also letzten Endes über Auflösungsvorsohriften, bei denen aber praktísch Branchbares und Unbrauchbares wabllos durcheinander geht. Dooh schon eine Sichtang der Methóden führt hier zu brauchbaren Ansätzen. Die Cauchysche Polygonmethode, die Methode der Näherungsfolgen (suocessive Approximation), die Uebertraguig der Simpsonschen Regel durch Runge, Heun und $\mathrm{K}$ utta sind die kurzen Namen der praktischen Verfahren, die wir nun fliichtig besprechen wollen.: Die sogen. Integration von

$$
\frac{d y}{d x}=f(x, y)
$$

darch geschiossene Ausdrücke ist in der Regel nicht möglich und erweist sich in den meisten Fallen, in denen sie theoretisch existiert, für die Praxis wie die Cardanische Formel als Fata morgana.

Die geometrische Bedeutung der Differentialglelohung (6) ist diese: Sie ordnet jedem Punkte in rechtwinkligen $(x, y)$-Koordinaten eine Rlchtung $f(x, y)$ zu, d. h. eine Gerade, deren Winkel mit der positiven $x$-Achse die trigometrische Tangente $f(x, y)$ hat. Die Differentialgleichung integrieren bedentet geometrisch: Man suche Karven, welche duroh jeden ihrer Pankte in der dort vorgeschriebenen Richtung hindurohgehen. Man findet somit eofort eine Näherung an die Integralkurve durch den Pankt. $\left(x_{0}, y_{0}\right)$, wenn man von $\left(x_{0}, y_{0}\right)$ ein Stïck in der Richtung $f\left(x_{0}, y_{0}\right)$ vorgeht, bis $\left(x_{1}, y_{1}\right)$, von da ein Stïck in der Riohtnng $f\left(x_{1}, y_{2}\right)$ bis $\left(x_{2}, y_{2}\right)$ usw. Man kann in manchen Fällen diese Zeichnung besonders bequem ausfuihren, indem man sich die Isoklinen $f(x, y)=$ konst. anfzeichnet und die zagehörigen Richtungen etwa in oiner "Windrose" anmerkt. Freilich wírd man so nur eine beschränkte Zahl von Isoklinen verzeichnen können und beim Zeichnen zwischen zwei Isoklinen die Richtung festhalten. So erhält man ein Polygon, welches als genaue İösung einer Differentialgleichung aufzufassen ist, deren Richtungsfeld zwischen zwei Isoklinen konstant ist und sich anf jeder Isokline sprunghaft ändert. Dementsprechend haben die Näherungspolygone Ecken. Dem kann man entweder dorch freihändige geeignete krumme Führung des Linienzuges zwischen zwei Isoklinen abbelfen oder man kann versuchen, die freihändig etwas unbequeme Richtangsinterpolation durch ein rationelles Verfahren zu ersetzen. Als besonders geeignet empfiehlt Runge zu diesem Zweck das Verfahren der Nhherungefolge. Sein Prinzip ist dieses: Es sei $y=y_{0}(x)$ das Naherungspolygon. Dann bestimme man eine weitere Näherangskurve, die durch $\left(x_{0}, y_{0}\right)$ geht, aus

Man setze also

$$
\frac{d y_{1}}{d x}=f\left(x, y_{0}(x)\right) \text {. }
$$

$$
y_{1}=y_{0}+\int_{x_{0}}^{x} f\left(x, y_{0}(x)\right) d x .
$$

Man kann dies Integral entweder rechnerisch bestimmen, nach irgend einem Verfahren der praktischen (mechanischen) Quadratur, oder man kann sich auch die Richtungskurve $y=f\left(x, y_{0}(x)\right)$ erst auftragen, um diese dann graphisch $z a$ integrieren. Bei deren Auftragen ist nun der Ort, wo man die Richtangsinterpolation durch eine bequemer zu handhabende Punktinterpolation ersetzt. Aus der so gefundenen Naherung $y_{1}$ bestimmt man analog eine zweite

$$
y_{2}=y_{0}+\int_{x_{0}}^{x} f\left(x, y_{1}(x)\right) d x
$$

asw. Dies Verfahren der Năherungsfolge konvergiert in der Regel außerordentlich rasch; namentlich wenn man sich nooh einiger aus der angeführten Literatur ersichtlicher VorsichtsmaBregeln bedient. Freilich sind nun unsere Literaturangaben noch durch den reichhaltigen Artikel von $R u n g e$ and Willers im zweiten Bande der mathematischen Enzyklopädie zo vervollständigen.

Eine andere, für Zeichnung und Rechnung gleich brauchbare Methode hat.Runge gefnnden, als er sich die Aufgabe stellte, die Simpsonsche Regel anf die Integration von Differentialgleichungen za übertragen. Dabel handelt es sich also um die Anfgabe, die Lösung $y(x)$ an der Stelle $x_{0}+h$ durch eina lineare Verbindung von Fanktionswerten 
10 zh approximieren, daB bei der Entwloklung des Naherungsausdruckes nach Potenzen yon $h$ möglichst viele Glieder mit der Entwloklung von $y\left(x_{0}+h\right)$ ubereinstimmen. Kutt a bat in Vervolletändigung der Rungeschen Ueberlegangen des Ergebnis'geínnden: DeberAntimmung bis zur vierten Potenz in $h$ erhält man, wenn man ansetzt

Hier ist

$$
y_{n}\left(x_{0}+h\right)=y_{0}+\frac{h}{6}\left(K_{1}+2 K_{2}+2 K_{3}+K_{1}\right)
$$

$$
\begin{aligned}
& K_{7}=f\left(x_{0}, y_{0}\right) \\
& K_{2}=f\left(x_{0}+\frac{h}{2}, y_{0}+\frac{E_{1} h}{2}\right) \\
& K_{3}=f\left(x_{0}+\frac{h}{2}, y_{0}+\frac{B_{2} h}{2}\right) \\
& K_{1}=f\left(x_{0}+h, y_{0}+K_{3} h\right),
\end{aligned}
$$

Zur Beurteilung der hiernach erreichten Genanigkeit finden sich in der Literatar mancherlei Angaben, denen ioh vom theoretischen Standpunkt mit einigem Miftranen bogegne. Immerhin labt sioh durob genaueres Eingeben auf die $R$ unge-Kuttasohen Rochnongen die folgende Fehlerabsohätzung gewinnen:

$$
\left|y\left(x_{0}+h\right)-y_{n}\left(x_{0}+h\right)\right|<\frac{6 M N\left|x-x_{0}\right|^{6} \mid N^{4}-1}{N-1}
$$

Hierbei coll fïr die in Betraoht kommenden $x$ - und $y$-Werte sein:

$$
\begin{gathered}
\left|x-x_{0}\right|<a,\left|y-y_{0}\right|<b \\
x-x_{0} \mid N<1 . \\
a M<b \\
|f(x, y)|<M \\
\frac{\partial^{(i+k)} f(x, y)}{\partial x^{i} \partial x^{4}}<\frac{N}{\Delta a^{k-1}} \quad(i+k \leqq 4) .
\end{gathered}
$$

Eln durobgerechnetes Beispiel findet man z. B. bei Sanden.

Die besprochenen Verfahren lassen sich leicht auf Systeme von Differentialgleiobungen ausdehnen. Damit beherrscht man dann natürlloh auch die gewöhnlichen Differentlalgleichungen hö̀herer Ordnung, wo toilweise noch besondere Methoden zur Verfügung ntoben, sowie die partiellen Differentialgleichungen erster Ordnung (Anfangswertproblem). Im Rungeschen und im Willersschen Buobe findet man auch das wenige für die Rand. wertalgaben bei elliptischen partiellen Differentialgleichungen zweiter Ordnung bisher Dekannte zusammengestellt. Ich gehe nicht näher darauf ein, da gerade in dieser Hinloht, wie icb höre, neue Veröffentlichungen berorstehen.

Es war der Zweck dieser Zeilen, für das Bekanntwerden dér neuen praktischen Mothoden 2a wirken. Wenn es mir gelungen ist, neues Interesse zu wecken, so wird auob weitere Verwendung der Methoden und nouer Fortschritt im Ausban der Methoden Alo Folge sein. Zu bedauern ist nur, daB -ein Lehrbuch groBen Stlls im Geblete der praktisohen Mathematik noch nicht vorliegt. Fin solches würde freilich neben dem hier dargestellten Hauptinhalt der vorliegenden Leitfaden nooh manches andere, z. B. manches aus der Praxis der Reihen usw. enthalten müssen.

\section{KURZE AUSZÜGE}

\section{Hydraulik und Hydromechanik.}

Energleverlust bel Querschnittserweiterund. Auf Veranlassurig und unter Leitung von Prof. Prandtl hat $\mathrm{Rich}$ a $\mathrm{rd} \mathrm{Kr} \delta$ nc: die Strómungsvorgãnge in slark erweilartę Kanälen untersuchl. (Versuche über strobmungen in stark erweiterten Kanālen. DisMartation, Berlin Techn. Hochsch., 1920, $85 \mathrm{~S}$; wueh Heft 222 der Fotschungsarbeiten a d
Gebiet des Ingenicurweseas, herausg. vom $\mathrm{V}$. d. I.) Die Querschnitte waren Rechtecke von gleichbleibender Hōbe $(254 \mathrm{~mm})$, deren Breite von rd. $68 \mathrm{~mm}$ auf rd. $251 \mathrm{~mm}$ bei verschiedenen Erweiterungswinkeln zunahm. Die prakLisch wichtigsten Ergebnisse, die der Aḅb. 67 der Dissertation zu entnehmen sind, lassen sich im AnschluB an dic in der Hydraulik übliche 